\title{
Analytical and Gravimetrical Studies of the Corrosion Inhibitory Behavior of Carnosine
}

\author{
Dhuha H. Fadhil \\ Department of Chemistry, College of Science, Al-Nahrain University, Baghdad-Iraq. \\ Corresponding Author: duhah.fadhel@gmail.com
}

\begin{abstract}
The corrosion inhibition characteristics of Carnosine (2-(3-aminopropanamido)-3-(1Himidazol-5-yl) propanoic acid) in corrosive solution for mild steel have been investigated at $30^{\circ} \mathrm{C}$ through gravimetrical technique namely Weight loss method. The morphology study of mild steel with and without Carnosine to impedance the corrosion for mild steel in $1.0 \mathrm{M}$ hydrochloric acid has been figured, utilizing with scanning electron microscopy (SEM). The results show that the investigated Carnosine might be an excellent corrosion inhibitor with enhanced inhibition efficiency of $91 \%$. The inhibition effectiveness of the Carnosine raising with increase of Carnosine concentrations, and decreases with increasing of temperature corrosion solution.
\end{abstract}

[DOI: $10.22401 /$ JNUS.21.3.10]

Keywords: Corrosion, Inhibitors, Carnosine, Gravimetry.

\section{Introduction}

Carnosine is kind of peptide which was found in the muscles skeletal in significance value [1]. These materials with a various of physio-logical function; they might capacity to lessen muscle exhaustion with enhance learning capacity due to their antioxidative impacts [2] and buffering limits because of the nearness of hetrocyclic gathering. Carnosine is a peptide made out of alanine and histidine and plays out different organic capacities, including $\mathrm{pH}$ buffering, layer securing movement, antioxidant, hostile to glycation, against maturing, bonding of divalent cationic metal with control of macrophage role [3]. Our inhibitor (carnosine) was a antioxidant of responsive species of oxygen that is generally disseminated in muscle with focal sensory systems [4]. The inhibitor was a part of potential in the treatment of extreme introvertedness [5], ulcers and diabetes illness [6]. An extensive variety of compounds have been effectively examined as effective corrosion inhibitors for the alloys and metals [7]. The best and productive inhibitor has been observed to act as a natural compound with $\pi$ bond, $\mathrm{N}, \mathrm{O}, \mathrm{S}$ and $\mathrm{P}$ atoms in addition to inorganic molecules, for example, chrome and nitro compounds [8]. Nonetheless, nearby testing of the studied molecules shows that most of them were poisonous while others are costly. These and numerous different elements have incited a proceeding with look for better inhibitors. MS (Mild steel) is a well-known iron type with numerous modern uses. It is described with significant mechanical quality and moderately minimal effort contrasted with other metal compounds. In any case, MS is very susceptible to molecules with MS corrosion haves involved awesome worry to different industries. Corrosive solutions, especially hydrochloric acid, are habitually utilized as a part of mechanical procedures, for example, corrosive cleaning, and corrosive pickling, with oil acidizing [9]. The corrosive solutions constitute solid destructive conditions for MS with accordingly; the investigation of the mild steel consumption is dependably a subject of high hypothetical and methodological. The utilization inhibitor was distinguished as an advantageous with inexpensive means of combat MS corrosion [10].

In this work Carnosine as shown in Fig.(1), was investigated as corrosion inhibitor of mild steel for the first time. Consequently, the inhibition characteristics of Carnosine were researched on the surface of MS in $1 \mathrm{M}$ hydrochloric acid employing analytical techniques namely weight loos and scanning electron microscope. The mechanism of inhibition has been studied on the basis of this investigation. 


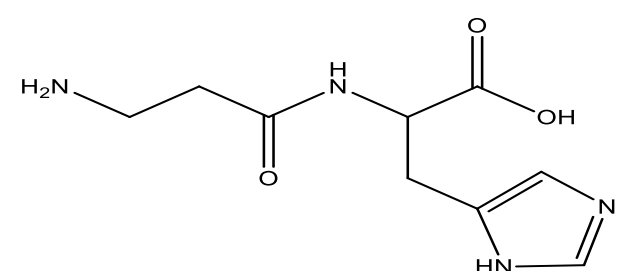

Fig.(1): Chemical Structure of Carnosine.

\section{Experimental}

\subsection{Chemicals and materials}

Solvents and acids were purchased from Sigma-Aldrch Company. Deionized distilled water has been utilized in the performed tests.

\subsection{Analytical Methods}

\subsubsection{Weight loss technique measurements}

Mild steel (MS) coupons with dimensions $2.0 \times 4.0 \mathrm{~cm}$ were abraded using various grades of emery papers, washed with acetone, rinsed with distilled water and dried utilizing filter paper. After weighting accurately, the coupons immersed in $100 \mathrm{ml}$ of $1 \mathrm{M} \mathrm{HCl}$. This methodology has been performed with and without various concentrations of Carnosine as corrosion inhibitor at $30^{\circ} \mathrm{C}$. Impacted of deferent times $(1,2,5,10 \mathrm{hrs})$ immersion was investigated. Each period of time mild steel coupons then washed with double distilled water, dried and weighted. The data of weight loss as in equation 1 , have been employee to calculate the corrosion Rate by equation (2):

$E \%=\frac{W_{1}-W_{2}}{W_{1}}$

where $W_{1}$ and $W_{2}$ were the losses weight of MS in gram without and with Carnosine

$R=\frac{\text { (weight loss in gram) } \times 8.75 \times 10^{4}}{D \times A \times T}$

where $\mathrm{D}$ is density of coupons of the investigated mild steel in $\mathrm{g} / \mathrm{cm} 3, \mathrm{~A}$ is area, $\mathrm{T}$ is time.

The coverage degree of the surface and inhibition efficiency was evaluated through equation 3 and 4 .

$\theta=\frac{C_{R}^{*}-C_{R}}{C_{R}^{*}}$

$E \%=\theta \times 100$

where $C_{R}^{*}$ corrosion rate with Carnosine and $C_{R}$ corrosion rate without Carnosine.

\section{Results and discussion}

\subsection{Gravimetric investigations}

\subsubsection{Impact of inhibitor concentrations}

Plots acquired from weight loss method for $\mathrm{MS}$ in $\mathrm{HCl}$ at the conc. of $1.0 \mathrm{M}$ in the corrosion solutions with and without of Carnosine at different conc. after inundation of MS for $1,3,5$ and $10 \mathrm{hrs}$ at $303 \mathrm{~K}$ the results shown in Fig.(2). The weight loss of MS in corrosive solution increments with time yet diminishes with expanding concentration of the tested compound demonstrating which performance of corrosion MS in corrosive solution is hindered by nearness tested comp. [11]. At significant temperature, it was characteristic that weight reduction of MS increments with raising temperature thus, Carnosine is adsorb on morphology of MS by mechanism of transfer from Carnosine to charged metal morphology, that underpins physical adsorption. Estimations of restraint performances and corrosion rate of Carnosine computed utilizing above equations have been displayed in Table 1

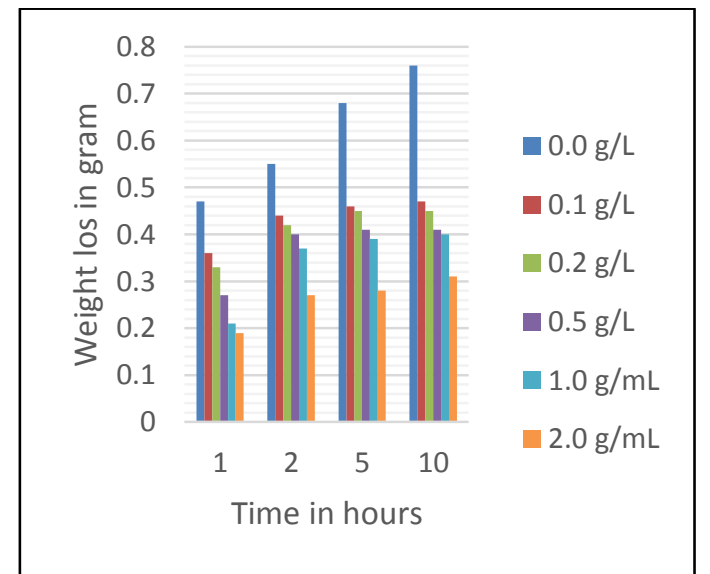

Fig.(2): Weight loss and times of MS in corrosive solution with and without of deferent Carnosine conc. at $303 \mathrm{~K}$.

The outcomes obtained and large revealed which the impedance performances of tested compound increments and increment in concentration, showing Carnosine act as adsorption compound. Impedance performances have found to diminish regarding to expanding temperature, that suggests the degree of adsorption additionally diminishes with increment in temperature. Thus the tested compound work via physical adsorption mechanism, that includes charge transfer from Carnosine to the surface of mild steel $[12,13]$. 


\subsubsection{Temperature Impacts}

In corrosive media, the rate of corrosion is affected by temperature change, by changing Arrhenius conditions, when CR was the rate of corrosion specified with the weight reduction estimation, Ea, the clear energy of activation, A was represent the steady of Arrhenius, R was (molar/gas) consistent whereas the absolute temperature is T. Clear active energies were resolved from the incline of logarithm of corrosion rate versus I/T diagram as in Fig.(3).

These significance values demonstrate that the with Carnosine increment, the $\mathrm{Ea}$ of the iron dis-solution reactions. The considered adsorption of Carnosine is expected to find at significant energies sites with Carnosine, which brings hindering of activation sites, should be related with rising of Ea of MS in the restrained state. The higher value of $\mathrm{Ea}$ with Carnosine compared to that in its nonappearance was interpreted as Physisorption reaction.

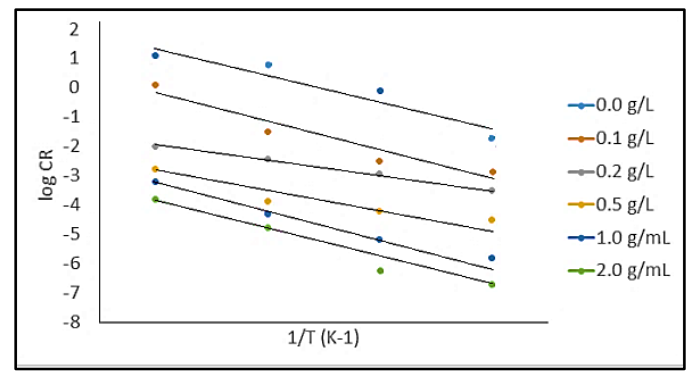

Fig.(3): Arrhenius plot for the corrosion of mild steel with various concentrations of Carnosine.

\subsection{SEM study}

Fig.(4) demonstrates the SEM pictures of MS after inundation in corrosive media in presence and absence of Carnosine. From the SEM image, the results exhibited that without Carnosine, the surface of the mild steel was genuinely eroded, exhibiting a fundamentally decayed morphology. Nonetheless, with the expansion of the inhibitor, the corrosion was clearly impedance as the harshness of the surface of the MS diminishing pointedly as per the pictures appeared in fig 4 . These outcomes additionally affirm that Carnosine may be used as a good performance inhibitor for MS corrosion at corrosive media.

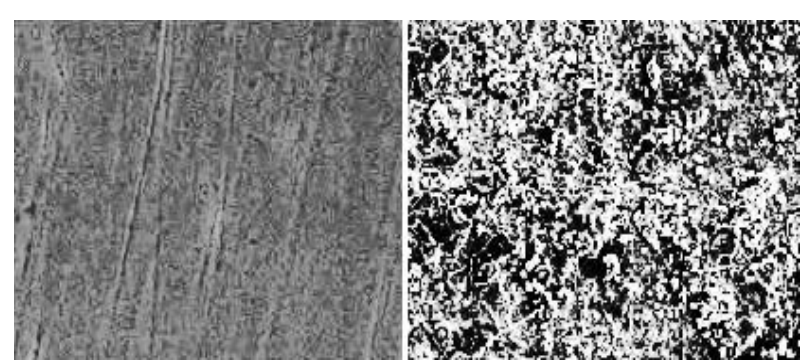

Fig.(4): The SEM images of MS morphology presence or absence of Carnosine.

\subsection{Conclusion}

The impact of corrosive solution and the impact of addition of Carnosine on the MS surface has been considered. The outcomes demonstrated that the corrosion performance of surface of the mild steel coupons is increment with expanding corrosion inhibitor concentration. The inhibitor showed a good behavior compared to other eco-friendly corrosion inhibitors to protect alloys from corrosive solutions. However, raising temperature results low inhibition performance but lowering temperature led to higher inhibition performance. Additionally, corrosion inhibitor characteristics of various types of surfactants could be conceivably utilized manufactures as great choice to take care of the issues of corrosion of metallic surfaces to keep the material and economic losses.

\section{References}

[1] Boldyrev, A.A. Carnosine and Oxidative Stress in Cells and Tissues; Nova Science Publisher, USA, (2006).

[2] Alpsoy, L. Akcayoglu, G. Sahin, H. Antioxidative and anti-genotoxic effects of carnosine on human lymphocyte culture. Hum Exp Toxicol., 30(12), 1979, 2011.

[3] Sale, C. Saunders B. and Harris, R.C. Effect of beta-alanine supplementation on muscle carnosine concentrations and exercise performance. Amino Acids, 39(2), 321.

[4] Chan K.M. and Decker E.A. Endogenous skeletal muscle antioxidants. Crit Rev Food Sci Nutr., 34(4), 403, 1994.

[5] Chez, M.G. Buchanan, C.P. Aimonovitch, M.C. Becker, M. Schaefer, K. Black, C. Komen, J. Child Neurol., J. 17, 833, 2002. 
[6] Hipkiss AR. Could carnosine or related structures suppress Alzheimer's diseas, J. Alzheimers Dis.,11(2), 229, 2007.

[7] Qiu S, Yu M, Liu L, Zhao H, Molecular dynamics and density functional theory study on relationship between structure of imidazoline derivatives and inhibition performance. Corr Sci, 50, 2021, 2008.

[8] Mu G, Li X, Qu Q, Zhou J. Molybdate and tungstate as corrosion inhibitors for cold rolling steel in hydrochloric acid solution. Corros Sci 2006 48, 445.

[9] Ulaeto, S.B.; Ekpe, U.J.; Chidiebere, M.A.; Oguzie, E.E. Corrosion inhibition of mild steel in hydrochloric acid by acid extracts of eichhornia crassipes. Int. J. Mater. Chem, 2, 158, 2012.

[10] Asegbeloyin, J.N.; Ejikeme, P.M.; Olasunkanmi, L.O.; Adekunle, A.S.; Ebenso, E.E. A novel Schiff base of 3acetyl-4-hydroxy-6-methyl-(2H)pyran-2one and 2,2'-(ethylenedioxy)diethylamine as Potential Corrosion Inhibitor for Mild Steel in acidic medium. Materials, 8, 2918, 2015.

[11] Dubey, A. K. and Singh, G. Corrosion inhibition of mild steel in sulphuric acid solution by using polyethylene glycol methyl ether (PEGME), Portugaliae Electrochimica Acta, 25(2), 221, 2007.

[12] H. Mohammed, O. Ahmed and A. A.AlAmiery, Synthesis and characterization of a novel organic corrosion inhibitor for mild steel in $1 \mathrm{~m}$ hydrochloric acid, In Press, Accepted Manuscript.

[13] Kumpawat, V., Garg, U. and Tak, R. K. Corrosion inhibition of aluminium in acid media by naturally occurring plant Artocarpus heterophyllus and Acacia senegal, Journal of Indian Council of Chemists, 26(1), 82, 2009. 